\title{
114. Ultrasonic Attenuation of the Brain in Acute Head Injury
}

\author{
Masuhisa OKa, Yasuaki NaIto, Nobuo OKu, Yasushi KujIRAoKa, \\ Keijiro Kiyoshima, Hitoshi HIgashiguchI, \\ Division of Neurosurgery, Department of Surgery, Osaka Labor-Disease \\ and Welfare Hospital, Sakai-City, Osaka \\ Katsuya Yoshioka and Akira OMura \\ Department of Ultrasonics, Research Institute of Industrial Science, \\ Osaka University, Hirakata-City, Osaka
}

Ultrasound is now in use by the authors for detecting intracranial hematoma and secondary induced cerebral changes of edematous states of injured brains. Clinical and experimental data of the authors on the ultrasonic attenuation of injured brains have revealed useful to detect appearing edematous states of cerebrum. The ultrasonic attenuation is observed on sampled thirty cases of acute head injuries in respect to changes of neurological and physical conditions. With the decrease of ultrasonic attenuation values there are cases of $46.7 \%$ with impaired clinical signs and with -7 $\mathrm{db}$ of attenuation decrease in mean value, and $16.6 \%$ of cases have shown improved neurological and physical signs. With the increase of ultrasonic attenuation values there are cases of only $8.7 \%$ with impaired clinical signs and with $+4 \mathrm{db}$ in mean attenuation value, and $56.7 \%$ of cases have showed improved neurological and physical signs.

Consequently it may be able to find the correlation between the ultrasonic attenuation and the neurological, physical signs. The decrease of ultrasonic attenuation is accompanied by symptoms of anisocoria, etc., i.e., signs of appearing edematous states of injured brains.

Ultrasonic beams for measuring the attenuation have to pass through the brain substance in large amount with little interference, so it is adequate to examine on the frontal bone outside to the edge of the frontal sinus and orbital ridge, inspecting the certain echo of the occipital bone on the same side. As there may be localized edema of injured brains, it may be neccessary to detect the fluctuaton of the ultrasonic attenuation of localized part of brains, i.e., temporal lobes and so far, by the distinct and non-uariable echo sources.

Clinical and experimental data above mentioned are confirmed by observing the absorpion coefficient of the brain. Normal brains of dogs show $0.61-0.64 \mathrm{db} / \mathrm{cm}$ on attenuation coefficient, injured brains show $0.41 \mathrm{db} / \mathrm{cm}$ in mean value and edematous brains induced by the i.e. injection of 
destilled water show $0.44 \mathrm{db} / \mathrm{cm}$. The difference found between these attenuation value is similar to the experimental and clinical data, and corespondingly to the decreased attenuation value edematous change in cerebral substance can be seen by the histologic examination.

\title{
115. Ultrasonic Attenuation of Brain and Brain Circulation-Ultrasonorheometry
}

\author{
Hisao Maruta, Hiroshi Hata, Genichi Suzuki, \\ Ikuo Yamaoka, Shigeki Kawasakı, Renzo Hirayama \\ and Jo INABA \\ 2nd Dept. of Surgery, Tokyo Medico-Dental College
}

\section{Ultrasonic Attenuation of Brain in Epilepsy (2nd Report): In Childfood Epilepsy}

\author{
Kenji InUgami, Hidehiko Hachrya, Akio Hayashi, \\ Matsuzawa Mental Hospital, Tokyo \\ Takechi Fujiwara, Matazo Kido \\ Umegaoka Mental Hospital, Tokyo \\ Toshio WaGaI, Shigeru Ishikawa and Kunio Yukishita \\ Juntendo University School of Medicine, Tokyo
}

Ultrasonic attenuation in brain tissue were examined on 46 cases of childfood epilepsy. Almost cases of these patients showed intense intelligence disorder such as imbecility (32 cases) and infant cases were combined with intense nervous disorder such as hemiplegia and unvoluntary movement too. Ultrasonic attenuation in brain tissue was measured using transmission method and a frequency of $2.25 \mathrm{Mc}$.

Measurements showed very interesting results that only 7 out of 46 case showed less attenuation and other 39 cases snowed large attenuation, particulary 16 cases out of them showed remarkable large attenuation such as more than $+25 \mathrm{~dB}$ compaired with normal brain. These results indicated the entirely reverse phenomena against the fact that the brain tissue of epilepsy in adult showed the tendency of less attenuation compaired with 